\title{
NOTES ON THE VARIETY OF MULTIPACTOR PATTERNS
}

\author{
Gennady Romanov, Fermi National Accelerator Laboratory, Batavia, IL, USA
}

\begin{abstract}
The experimental results on multipactor often are not in a good agreement with theoretical predictions and numerical simulations. The experimental results can noticeably vary from test to test even in the same geometry of electrodes, depending on the material they made of and condition of their surfaces, i.e., secondary emission properties. On the other hand, an actual secondary emission yield (SEY) of electrode material is never known a priory in the experiments. In practice, the SEY data for given material used for multipactor evaluation are obtained in specialized measurements. A difference between actual and tabulated SEY of material may result in a disagreement between predictions and measurements. In this work the impact of the basic SEY characteristic variations on the multipactor dynamics in a variety of coaxial and rectangular waveguides is studied. The study was performed numerically with the use of CST Particle Studio.
\end{abstract}

\section{INTRODUCTION}

In general, the multipactor phenomenon is discussed in terms of resonance between the oscillations of the electrons and the RF electric field in the space between electrodes of $\mathrm{RF}$ device. When resonance theory is used to analyse multipactor, it predicts a growth of an electron avalanche only within relatively narrow separated intervals of RF field strength. However, in the experiments the multipactor is observed often within a wider range of field levels with the overlapping (merging) of multipactor zones. It was found that spread of initial velocities of secondary electrons, space charge effect, elastic and diffused scattering being considered may noticeably widen and change character of the predicted multipactor bands $[1,2]$. But the reason for discrepancy between multipactor predictions and experiments is not that these parameters are unknown. The general secondary emission properties (SEY) of many common materials are well studied and documented. The reason is that the emission parameters are very sensitive to the current surface condition of the electrodes, and therefore the exact SEY properties are never known a priory in each practical case.

A very illustrative example of such uncertainty can be found in the experimental work [3], where multipactor bands being measured in the same experimental setup changed significantly for the same material depending on the history of storage and treatment (see Tab.1). The picture was even more diverse when MP intensity and processing time were considered. Besides that, the SEY parameters affect differently in the different geometries and
RF field configurations. For example, initial velocity of secondary electrons produces negligible effect in the narrow gaps between electrodes, but dramatically changes character of multipactor from resonant to non-resonant one in the big gaps [4]. Reflected waves in the waveguides also can create confusing picture of multipactor, especially if actual standing wave ratio is not known accurately enough or it changes unpredictably.

Table 1: Some MP bands of $1^{\text {st }}$ order from [3]

\begin{tabular}{|l|c|}
\hline \multicolumn{1}{|c|}{ Material } & MP band, $\mathbf{~ k V} / \mathbf{m}$ \\
\hline Copper & $132.1-188.4$ \\
\hline Copper baked at $400^{\circ} \mathrm{C}$ & $145.8-231.4$ \\
\hline Copper stored one week in PE bag & $139.0-192.8$ \\
\hline TiN on copper & $129.3-170.7$ \\
\hline Copper electroplated on steel & $132.7-183.8$ \\
\hline
\end{tabular}

In the present work the impact of the basic SEY characteristic variations on the multipactor dynamics in a variety of coaxial and rectangular waveguides at different powers and standing wave ratios is studied. The observed patterns explain some experimental multipactor data that sometimes was not quite understandable. The study was performed numerically with the use of CST Particle Studio. In present simulations we followed our practical approach described in detail elsewhere [5].

\section{SECONDARY EMISSION FUNCTIONS}

A quantity of secondary electrons in these models depends only on the energy of primary incident electrons $\mathrm{W}$ and angle of collision. Therefore, the impact of elastic and diffusion scattering on the MP dynamic is missed here. The following secondary emission yield functions SEY(W) were used in this work.

First, the analytical (based on Vaughan model) SEY functions with equal maximal values $\mathrm{SEY}_{\max }$, but different locations of maximums $\mathrm{W}_{\mathrm{m}}$ : $200 \mathrm{eV}(1), 300 \mathrm{eV}$ (2) and $400 \mathrm{eV}$ (3). (shown in Fig.1). Additionally, four true SEY functions from CST library were used - the functions are shown in Fig 2. All SEY functions have different energy of collision $\mathrm{W}_{1}$ and $\mathrm{W}_{2}$ at which $\mathrm{SEY}=1$ (first and second crossovers). The complete Vaughan model also includes the offset $\mathrm{W}_{0}$ (there is no emission for $0<\mathrm{W}<\mathrm{W}_{0}$ ), but it was not used in the study. For quick reference the SEY function main parameters are organized in the Table 2.

All SEY functions were used with different probability density functions (PDF) of initial energy of secondary electrons. Three PDFs with most probable values of initial secondary electron temperature $\mathrm{T}_{\mathrm{e}}$ of $0.1 \mathrm{eV}, 5 \mathrm{eV}$ and $7.5 \mathrm{eV}$ 
(latter is CST default value in SEY models) are shown in Fig.3. All curves in Fig.1-3 are plotted for $0^{\circ}$ incident angle of primary electrons.

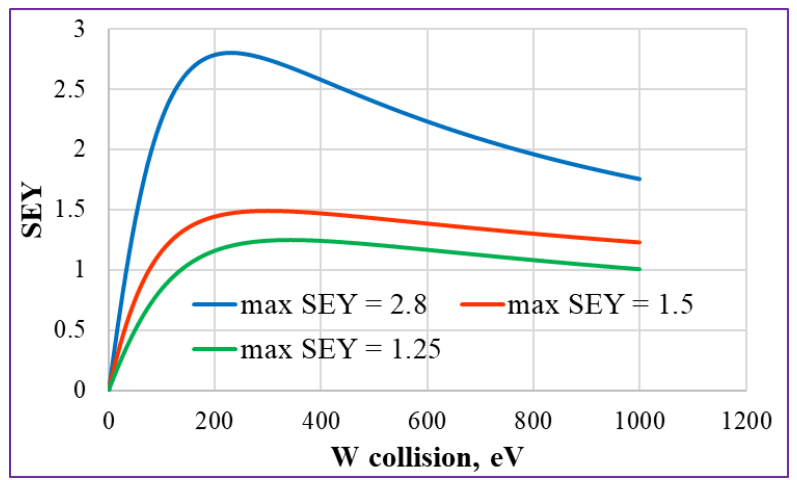

Figure 2: SEY functions from CST library

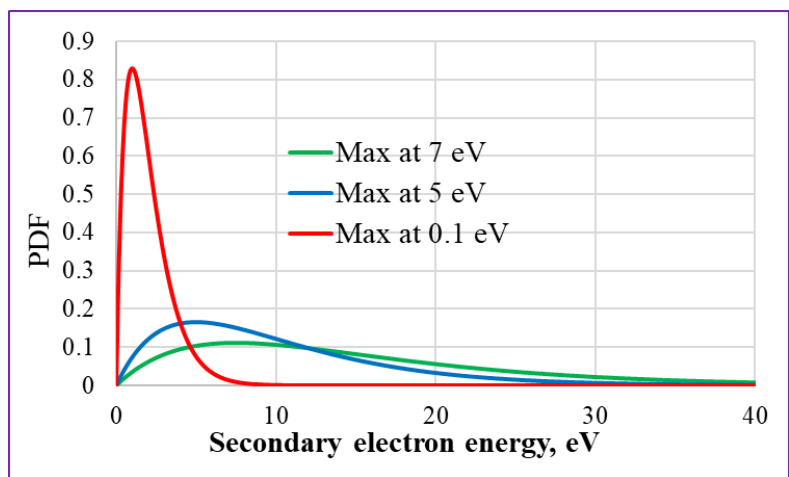

Figure 3: Probability density functions used in the simulations.

Table 2: Imported "true" SEY functions

\begin{tabular}{|l|l|l|l|l|}
\hline data ID & $\mathbf{S E Y}_{\max }$ & $\mathbf{W}_{\mathbf{m}}, \mathbf{e V}$ & $\mathbf{W}_{\mathbf{1}}, \mathbf{e V}$ & $\mathbf{W}_{\mathbf{2}} \mathbf{e V}$ \\
\hline $1.49 @ 200$ & 1.49 & 200 & 51 & 1250 \\
\hline $1.49 @ 300$ & 1.49 & 300 & 77 & 1600 \\
\hline $1.49 @ 400$ & 1.49 & 400 & 102 & 2000 \\
\hline $2.8 @ 230$ & 2.8 & 230 & 33 & 2000 \\
\hline $1.9 @ 277$ & 1.89 & 277 & 61 & 1750 \\
\hline $1.5 @ 300$ & 1.5 & 300 & 77 & 1900 \\
\hline $1.2 @ 291$ & 1.25 & 291 & 137 & \\
\hline
\end{tabular}

\section{MULTIPACTOR BETWEEN PARALLEL PLATES}

\subsection{Model}

Parallel plates are the simplest configuration in which MP can arise, and it is used for MP theory development since pioneering works [6,7]. Many theoretical and experimental works on MP between parallel plates make this configuration a very convenient example for study and analyses. It should be mentioned that in real rectangular waveguides MP dynamic is more complicated due to the transverse variation of RF fields [8].

A model for simulations is simple - it consists of two metal rectangular plates separated by variable gap; the size of the plates is two times gap distance (arbitrary choice). The fields between plates are uniform and were calculated in external CST TD (time domain) project and imported into CST PIC solver.

For the field calculations with TD solver, the model was equipped with two ports, and the boundary conditions were defined as shown in Fig.4, providing uniform transverse field distribution. With two ports in TD, it is possible to simulate field of any standing wave ratio which is important especially for long models. But for the short models, in which the plate lengths are much shorter than the wavelengths under interest, standing wave ratio is almost undistinguished and only field amplitude matters. So, as far as the models were short like this, only pure traveling wave regime was used. Elementary MP theory does not consider magnetic field, so only electric field was imported for simpler comparison, distribution of which is shown in Fig.5.

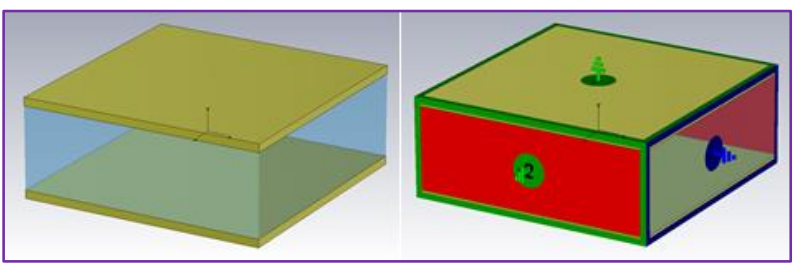

a)

b)

Figure 4: a) The model geometry. b) The model boundaries in TD solver are green - electric wall, blue - magnetic wall, red - waveguide port.

However, in the long structures, considered later, TD solver built in PIC solver was used, because it is more effective in generating a variable standing wave ratio.

Conveniently, CST PIC solver has an option of time dependent source of initial particles, which allows distributing the initial particles over phases of RF fields. We used "Particle Area Source" with Gaussian emission model, which seems to be the most flexible and handy for MP simulations. The details on Gaussian particle source setting and PIC solver setting in general are given in [9].

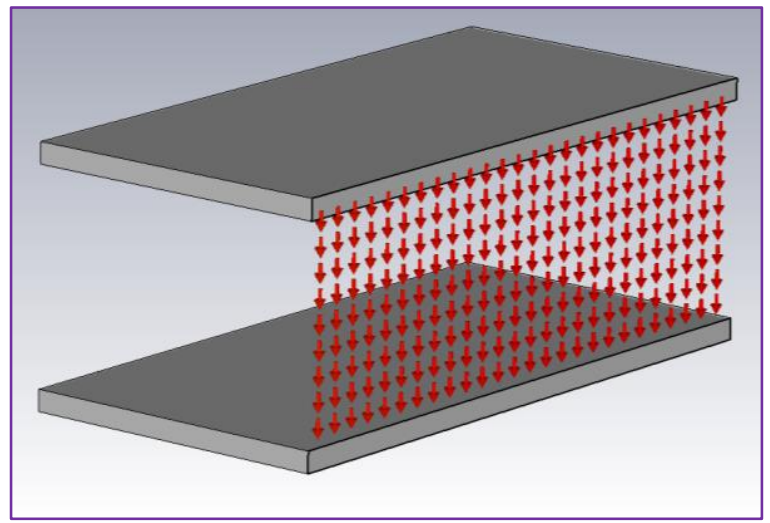

Figure 5. Electric field distribution in the parallel plates model.

\subsection{Multipactor without space charge}

The simulations of MP without space charge and zero initial energy of secondary electrons $\mathrm{T}_{\mathrm{e}}$ were performed just as a benchmarking and for comparison with elementary theory formulas. 
The parameters for the model were taken from experimental work [3], namely: $10 \mathrm{~mm}$ gap between plates and $500 \mathrm{MHz}$ frequency of RF electric field between the plates. The resonant condition for two side multipactor between parallel plates is given by the elementary theory formulae:

$$
\begin{aligned}
\text { Voltage } & =\frac{m_{e}}{e} \cdot \frac{4 \pi f^{2} d^{2}}{2 n-1}[\text { Volts }] \\
\text { or } & \text { E - field }=\frac{m_{e}}{e} \cdot \frac{4 \pi f^{2} d}{2 n-1}[\mathrm{~V} / \mathrm{m}],
\end{aligned}
$$

where $\mathrm{f}[\mathrm{Hz}]$ is field frequency, $\mathrm{d}[\mathrm{m}]-$ gap between plates, $\mathrm{m}_{\mathrm{e}}[\mathrm{kG}]$ - electron mass, $\mathrm{e}[\mathrm{C}]$ - electron charge, $\mathrm{n}$ - order of multipactor $(1,2,3 \ldots)$.

The two-side MP according to the equations (1) is expected at the voltages $1786 \mathrm{~V}(\mathrm{n}=1)$ and $595 \mathrm{~V}(\mathrm{n}=2)$. The MP of third order $(n=3)$ would be at voltage of $357 \mathrm{~V}$, but MP of this and higher modes cannot develop because of low energy of collision. There are more sophisticated formulae that consider initial energy of secondary electrons, energy of collision etc, and they can define MP bands, not just resonance lines $[7,10]$. Though, the current simulations with zero emission energy and without space charge effect are not for detailed comparison with these advanced theories - they are just a reference point for following more complex simulations.

In these initial simulations the material of plate had emission properties SEY 2.8@230 (wet treated Nb). The initial energy of secondary electrons was set zero $\mathrm{T}_{\mathrm{e}}=0$, as it is in the elementary theory. Voltage between the plates was swept from $300 \mathrm{~V}$ to $3.5 \mathrm{kV}$ with pre-defined step. At each voltage level a bunch of electrons was injected in the gap between plates and particle tracking was performed. The process of tracking stopped if an exponential growth of secondaries was detected, or simulation time limit was exceeded. In both cases an effective secondary emission yield was calculated by:

$$
<\text { SEY }>=\frac{\text { Emission current }}{\text { Collision current }},
$$

where corresponding currents are averaged over last several RF periods. The value of $<\mathrm{SEY}>$ greater than 1 indicates multipacting process and being compared to the maximal value of SEY function of material evaluates how "good" dynamic conditions are for MP. Sometimes exponential growth rate of number of particles $\alpha$ is used to indicate MP process. Effective secondary emission yield $<$ SEY $>$ can be converted to this parameter via expression $<$ SEY $>=\mathrm{e}^{\alpha^{*} \mathrm{RF} \text {-period }}[9]$. It should be noted that both parameters do not characterize the intensity of MP process, they only indicate particle multiplication and its rate.

The result of simulation is shown in Fig.6. and compared to predictions of elementary theories. The simulations without space charge effect are convenient to show pure impact of secondary electron initial energy spread. Therefore, the result of simulations with $\mathrm{T}_{\mathrm{e}}=7.5 \mathrm{eV}$ is also shown in Fig.6 to compare with. The result of comparison is well known from literature: the monochromatic emission pro- duces narrow barriers, while the emission with initial energy spread produces wider merged resonances shifted to lower voltage.

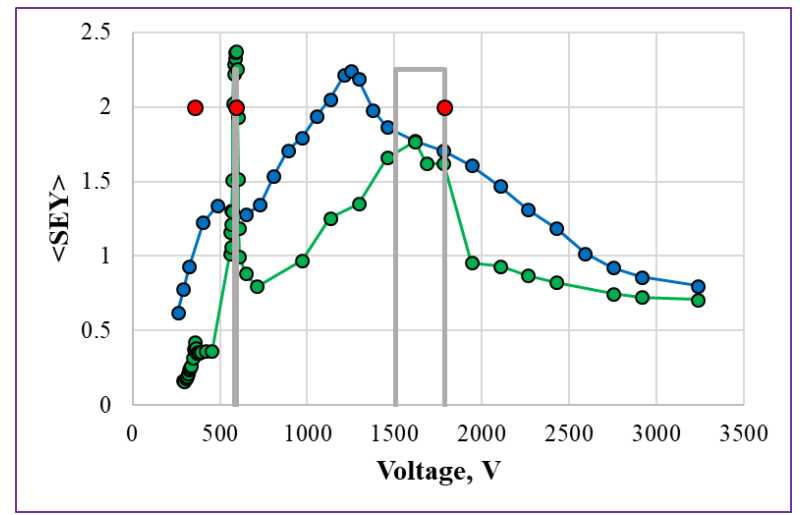

Figure 6: Effective secondary emission yield $<$ SEY $>$ obtained with different models without space charge effect: red dots are resonant conditions from (1); green line- simulations with $\mathrm{T}_{\mathrm{e}}=0$; blue - simulations with $\mathrm{T}_{\mathrm{e}}=7.5 \mathrm{eV}$; grey boxes are MP bands from [7], $\mathrm{T}_{\mathrm{e}}=0$.

\subsection{Multipactor of 1st order with space charge}

Developed multipactor is essentially a space charge limited process, and its first phenomena is a saturation of the discharge current density or number of particles as shown in Fig.7 (simulation were done with SEY 1.49@200 and $\mathrm{T}_{\mathrm{e}}$ $=7.5 \mathrm{eV})$ ). During developed two side multipacting there are one or several bunches of electrons in between the plates (number depends on MP order), which are well formed by phase focusing mechanism. Space charge of an electron bunch pushes peripheral particles out from phase stability interval (and possibly from area where dynamic conditions for multipactor exist). Therefore, some part of electrons continuously goes out of the game every RF cycle. This loss of electrons is compensated by secondary electrons that are emitted at each RF cycle. Finally, a dynamic equilibrium is established between losses and emission. The process comes to the steady state regime in which discharge current density stops at certain level, and no infinite exponential growth of particle number occurs (assuming that power source limitation, mismatching, RF cavity detuning etc do not break MP process). Current density at saturation has a fundamental limit that in general can be evaluated [11, 12, 13, 14]. Therefore, a total emission current is a natural parameter to evaluate and compare the intensities of MP barriers

Direct comparison of multipacting intensity with and without space charge effect is not possible. Growth rate $\alpha$ in saturated regime is zero, therefore it cannot be an indicator of multipacting at all. Effective secondary emission yield $\langle$ SEY $>$ is not a convenient indicator either since it always equals 1 at multipacting saturation regardless intensity of discharge $\left(\mathrm{I}_{\mathrm{coll}}=\mathrm{I}_{\text {emis }}\right.$ at saturation). Instead, a total steady state emission current was used as characteristic and measure of MP in case of active space charge effect and compared with effective secondary emission yield $\langle$ SEY $>$ 
obtained in simulation without space charge. The comparison resulted in the MP emission current and $\langle\mathrm{SEY}>$ as functions of voltage between plates is shown in Fig.8.

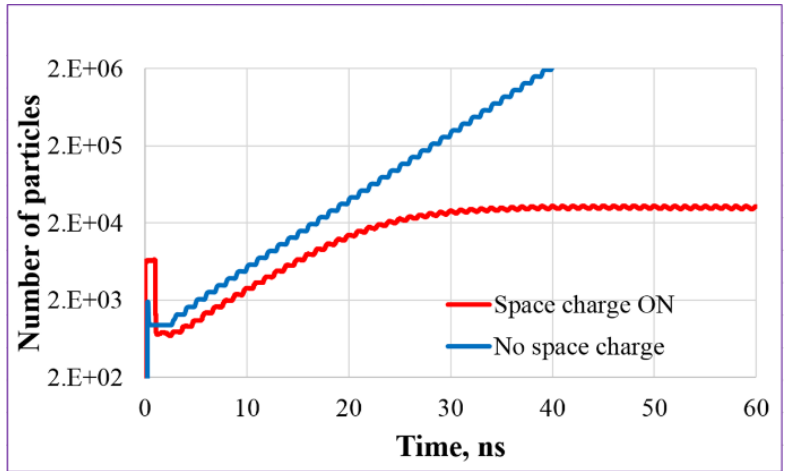

Figure 7: Saturation of number of particles in the PIC simulations of multipactor with space charge ON compared to the simulations in the same model without space charge effect (vertical scale is logarithmic).

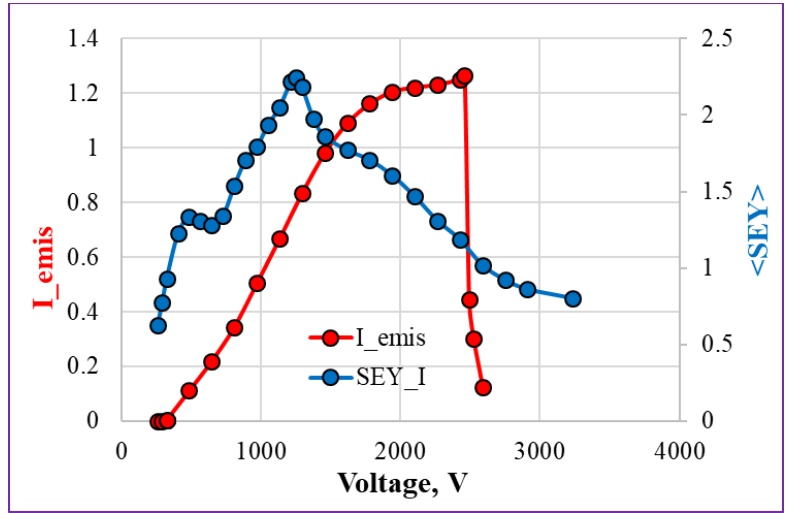

Figure 8: Comparison of first order MP bands simulated with (red) and without (blue) space charge effect. SEY of material is 2.8@230, $\mathrm{T}_{\mathrm{e}}=7.5 \mathrm{eV}$ for both simulations.

One more noticeable feature of space charge limited multipacting shown in the simulations and found in the experiments $[9,15]$ is that the energy of collision of particles with plates (or device walls) is much lower than what the elementary theory predicts. The average energies of collisions with and without space charge can be compared directly and are shown in Fig.9. Low energy of collision is qualitatively understandable. Effective secondary emission yield is equal to unit at MP saturation. So, the energy of collision tends to be higher than $\mathrm{W}_{1}$ just enough to compensate particles losses due to the debunching. The space charge related mechanisms that explain this decrease of the energy of collisions against metal are discussed in $[12,16]$.

A typical effect of the space charge is that MP bands simulated with space charge effect are shifted toward higher field levels compare to the ones simulated without space charge. Above upper boundary of the MP barrier multiplication of particles stops, space charge field disappears, and average energy of collision of remaining non-resonant particles jumps up abruptly due to higher voltage.

Impact of maximal value of SEY. Impact of maximal value of secondary emission function of plate material on
MP dynamic is rather obvious - the multipacting is more intense and MP band is wider for higher SEY of material (see Fig.10).

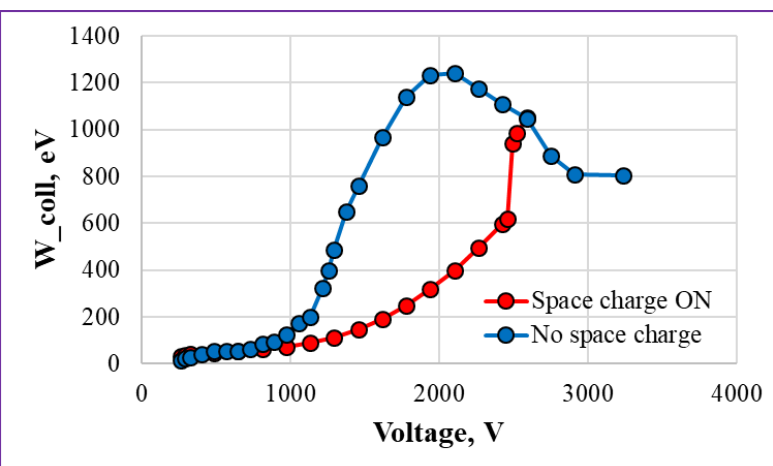

Figure 9: Comparison of energy of collision in MP band of first order.

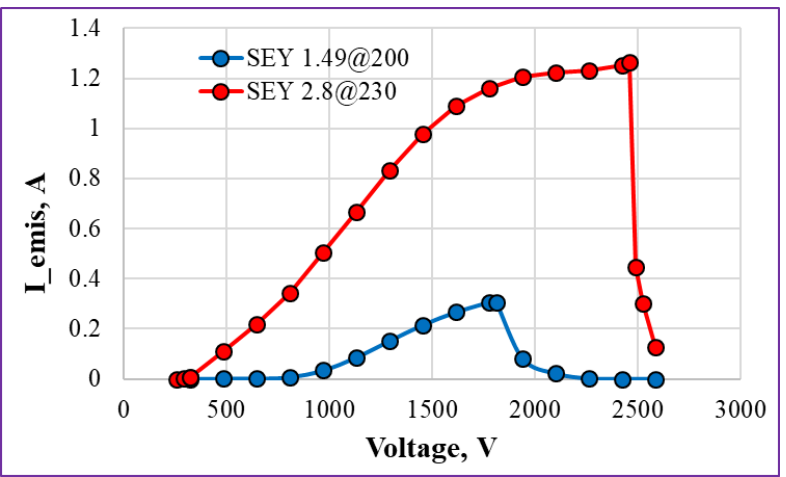

Figure 10: MP bands for materials with different maximal secondary emission and equal $\mathrm{T}_{\mathrm{e}}=7.5 \mathrm{eV}$.

Impact of location of max SEY. To see this impact the simulations were performed with secondary emission functions1.49@200,1.49@300, and 1.49@400 eV. All three functions were used with $\mathrm{T}_{\mathrm{e}}=0.1 \mathrm{eV}$ to clear the picture from effect of initial energy spread of secondary electrons. The simulated MP barriers are shown in Fig.11.

Emission current is higher, and the band is wider for plate material with location of maximal SEY at higher collision energy, since the range of favourable collision energies $\left(\mathrm{W}_{1}-\mathrm{W}_{2}\right)$ is larger as it can be seen from Table 1 .

Impact of initial energy of secondary electrons. The simulations performed with different spread of initial energy of secondary electrons $\left(\mathrm{T}_{\mathrm{e}}=0.1,1 \mathrm{eV}\right.$ and $\left.7.5 \mathrm{eV}\right)$ just illustrate earlier findings ([17] for example) that an increase of the spread of initial velocities results in overlapping of the multipactor zones and widening of a barrier, if the secondary emission is high enough - results in Fig.12 were obtained with SEY 2.8@230. If not, the higher multipactor zones may not be seen at all (See again Fig.11).

Frequency-gap product $\mathbf{f} \cdot \mathbf{d}$ is very important scaling parameter, which is used as an argument in many analytical models and susceptibility charts. When it comes to quick evaluation of a width of MP barrier this parameter proved to be handy and accurate enough [18]. The simulations of MP between parallel plates with fixed $f \cdot d=500 \mathrm{MHz} \cdot \mathrm{cm}$, 
but variable $f$ and $d$, result in practically the same MP barrier. It means that only one combination of frequency and gap distance can be studied or measured to find MP barriers at given voltage for other combinations, which is convenient for RF design.

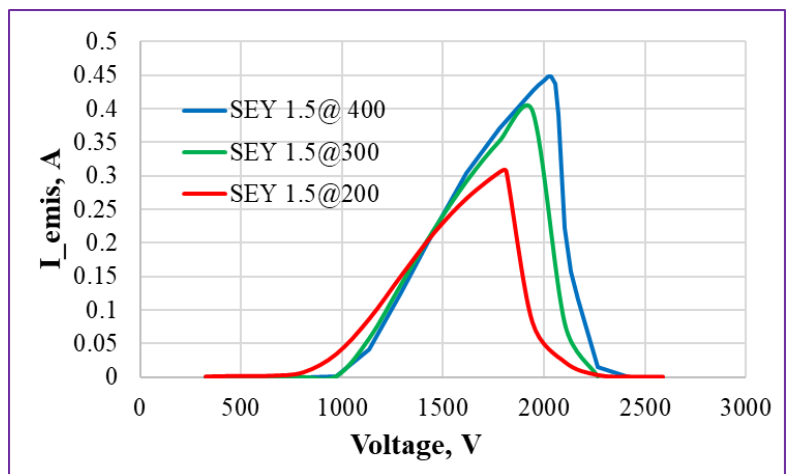

Figure 11: MP bands for plate materials with different locations of max SEY and the same $\mathrm{T}_{\mathrm{e}}=0.1 \mathrm{eV}$

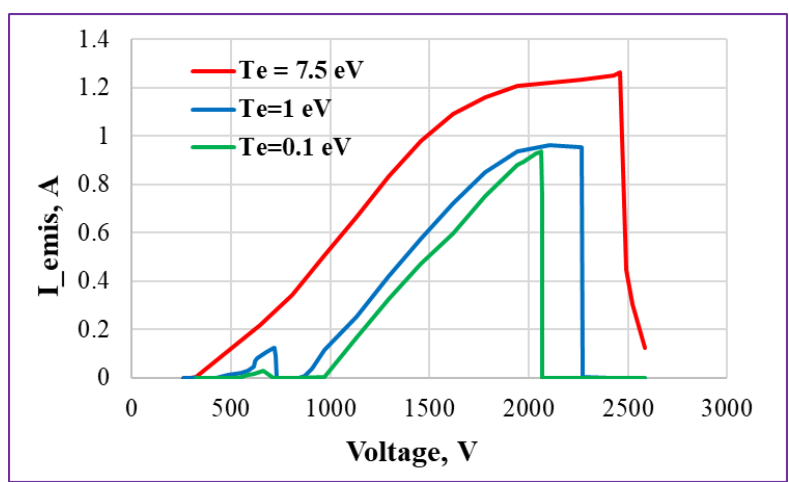

Figure 12: MP bands for plates made of the materials with the same SEY 2.8@230, but different spread of initial energy of secondary electrons. For more monochromatic emission MP resonance of second order is seen separately from first order resonance.

Intensity of multipactor decreases with gap distance increase because more particles go out of stable phase motion interval on their way from plate to plate due to the initial velocity spread and space charge effect.

However, the scaling is not applicable to the non-resonant multipacting [4], which is not considered here.

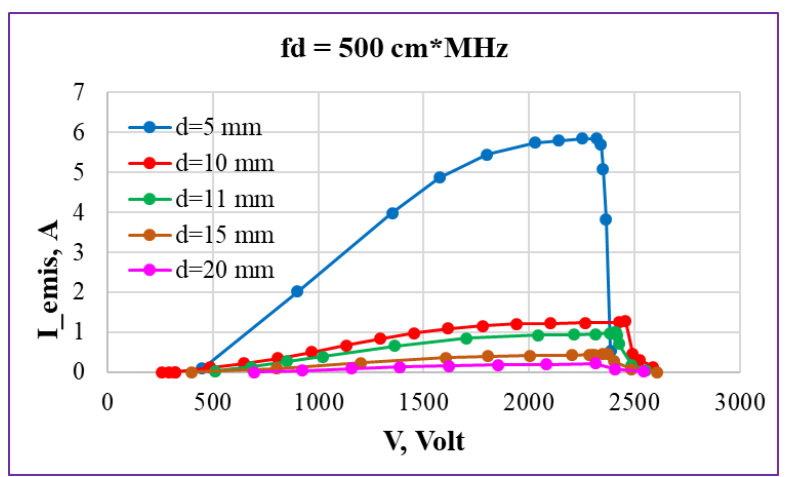

Figure 13: MP barriers in the models of parallel plates with constant frequency-gap product, material of plates is SEY 2.8@230, $\mathrm{T}_{\mathrm{e}}=7.5 \mathrm{eV}$.
Impact of variable standing wave ratio. Standing wave may appear in the transmission lines due to mismatching between power source and load, non-uniformities in the lines or during normal transition regimes. Variable reflection coefficient is defined here as $\rho=\mathrm{A}^{-} / \mathrm{A}^{+}$, where $\mathrm{A}^{-}$and $\mathrm{A}^{+}$are amplitudes of reflected and incident waves respectively, and $\rho$ can change from 0 (pure TW) to 1 (pure SW). Amplitude of the superimposed field in each point of the transmission line depends on $\rho$ and phase of reflected wave. Complete distributions of the superimposed field amplitude can be seen in the half-wave long model (see Fig.14).

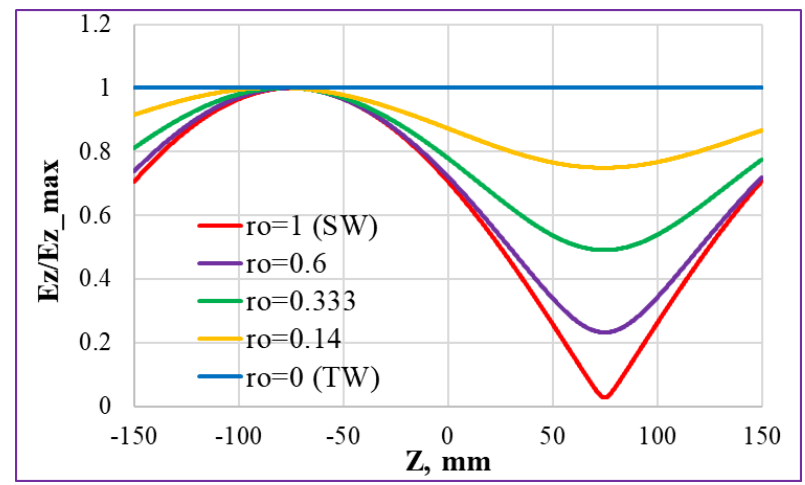

Figure 14: Distributions of normalized amplitude of superimposed wave at different reflection coefficients and fixed phase of reflected wave.

Simulations of multipactor in the half-wave long model show that familiar shape of MP barrier at $\rho=0$ gradually distorts and expands toward higher voltage with growth of reflection (see Fig.15). As a result of that the MP barriers become much wider, though less intense.

The plots in Fig. 16 explain the mechanism of this transformation for $\rho=0.333$ and incident wave amplitude $\mathrm{A}_{0}{ }^{+}=$ $865 \mathrm{~V}$ (the parameters were chosen as a convenient example). The maximum of non-uniform voltage distribution at $\mathrm{A}_{0}{ }^{+}$reaches multipactor level of first order. Simultaneously the minimum of voltage distribution at this incident wave amplitude is still at multipactor level of second order. This mix of orders in long structures is typical for $\rho$ closer to unit and for pure standing wave but it does not necessarily start exactly at $\rho=0.333$-it depends on given value of $f \cdot d$ ).

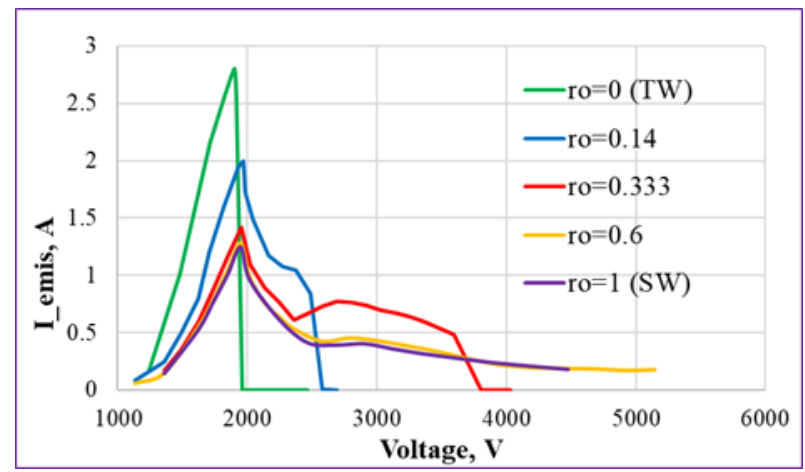

Figure 15: MP barriers in half-wave long model of parallel plates as functions of incident wave voltage at different reflections $\rho$. 


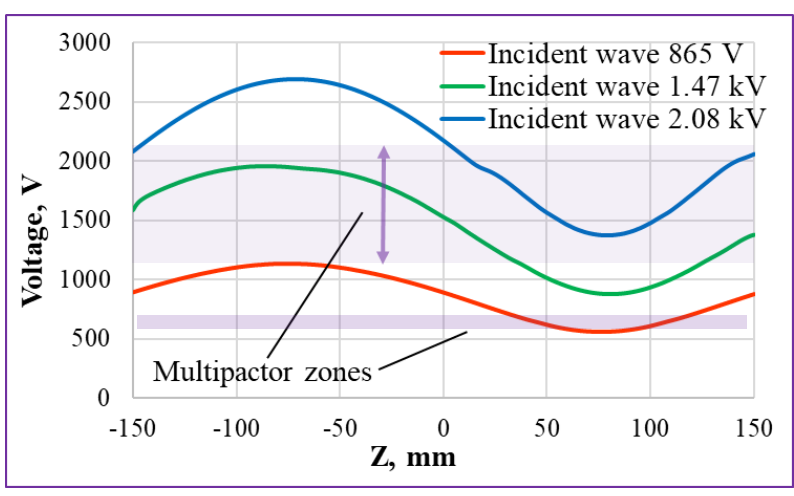

Figure 16: Crossing of the multipactor zones by voltage distribution at $\rho=0.333$ with incident wave amplitude $\mathrm{A}_{0}{ }^{+}=865 \mathrm{~V}, 1,47 \mathrm{kV}$ and $2.08 \mathrm{kV}$. The semi-transparent violet bands indicate multipactor zones of 1 st and 2nd orders according to Fig. 12.

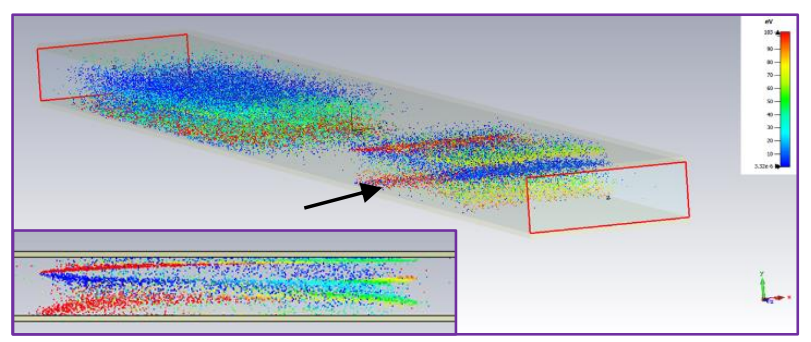

Figure 17: The snapshot of multipacting in the halfwave long model at amplitude of incident wave $\mathrm{A}_{0}{ }^{+}=865 \mathrm{~V}$ and $\rho=0.333$. Colour of the particles indicates their energy from 0 (blue) to $100 \mathrm{eV}$ (red). The insert in left bottom corner shows a sideview of 2 nd order multipacting

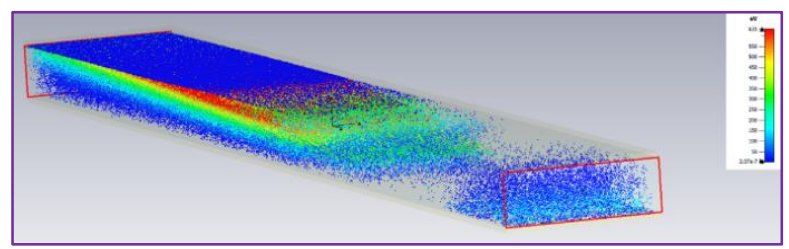

Figure 18: The snapshot of multipacting in the halfwave long model at amplitude of incident wave $1.47 \mathrm{kV}$ and $\rho=0.333$. The voltage along the model is almost completely inside multipactor zone of $1^{\text {st }}$ order (see also Fig.16).

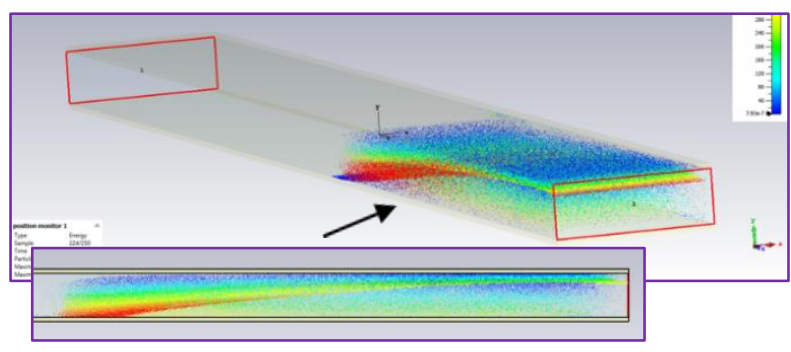

Figure 19: The snapshot of multipacting in the halfwave long model at amplitude of incident wave $2.08 \mathrm{kV}$ and $\rho=0.333$. The voltage is partially inside multipactor zone of 1st order (see Fig.16). The insert in left bottom corner shows a sideview of $1^{\text {st }}$ order multipacting in this part of the model (compare with Fig.17).
The snapshot of multipacting at $\mathrm{A}_{0}{ }^{+}=865 \mathrm{~V}$ is shown in Fig.17. Further increasing of the incident wave moves the voltage levels almost completely into MP zone of first order, and multipacting intensity is the highest (Fig.18). Finally, the minimum of the voltage distribution crosses MP zone, and the $2^{\text {nd }}$ order multipacting in this part of the model is replaced with multipacting of first order. The snapshot of this multipacting is shown in Fig.19.

\subsection{Multipactor modes of high order}

For simulation of high order multipacting the model has been changed per the experiments in [6]. In these experiments four multipactor modes were observed in the big $25.4 \mathrm{~cm}$ gap at frequency $50 \mathrm{MHz}$ with impressive resolution: $1 \mathrm{st}$ order at $10 \mathrm{kV}, 2$ nd order at $3.5 \mathrm{kV}, 3$ rd order at 2 $\mathrm{kV}$ and 4 th at $1.1 \mathrm{kV}$ (probably mixed with $5^{\text {th }}$ order).

In a big scale picture, the agreement between the experiments, elementary theory predictions, simulations with and without space charge is good as shown in Fig.20 (except a resonance at $\approx 6 \mathrm{kV}$, which will be discussed later). The energy of collision in each MP barrier is almost equal in simulations with and without space charge effect. It contradicts to the result obtained for $10 \mathrm{~mm}$ gap (see Fig.9). This inconsistency is not fully understood.

The low emission current of high order multipacting modes attracts attention. Intensity of high order modes are lower in principle due to the narrower intervals of phase stability, but there may be one more reason. The high order modes simultaneously have $2 \mathrm{n}-1$ bunches of particles (or sheets, $\mathrm{n}$ - mode number) in a gap (see Fig. 21 for example). Maybe, the complicated space charge field additionally suppresses re-emission (or reduce phase stability or both) and reduce total current.

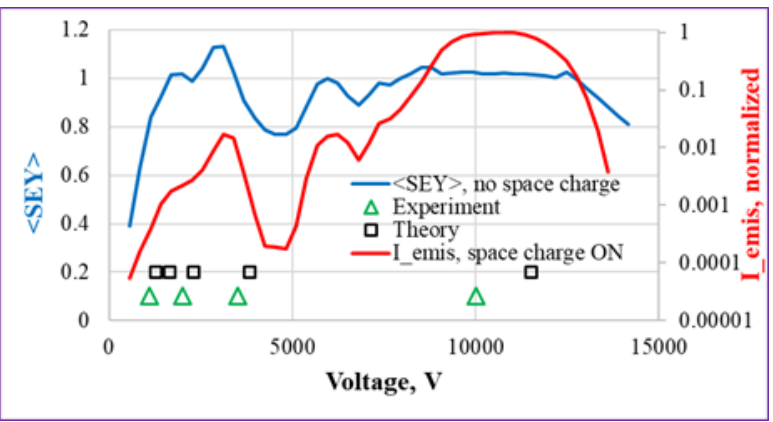

Figure 20: The barriers of multipactor in $25.4 \mathrm{~cm}$ gap simulated, measured, and predicted by elementary theory.

The multipacting mode at $\approx 6 \mathrm{kV}$ is due to the existence of a peculiar resonance trajectories, described in [19]. These trajectories have very long time of flight $\left(\approx 550^{\circ}\right)$ and therefore have very narrow interval of phase stability. The stable phase motion along these trajectories can exist just in case of very monoenergetic emission or sufficiently high SEY. That is why this mode was not observed in the experiments. In the simulations with Furman-Pivi emission models in which maximal SEY $<2$ this mode was not observed either. 


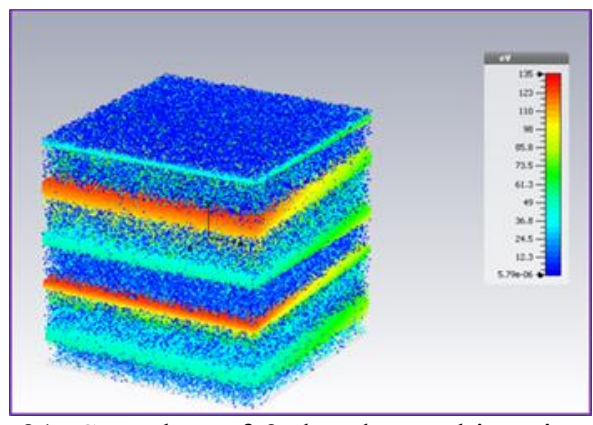

Figure 21: Snapshot of 3rd order multipacting mode. Five "sheets" of particles are clearly seen. Particle colours indicate their energy.

\section{MULTIPACTOR IN COAXIAL LINES}

The simulated multipactor bands in coaxial transmission lines and experimental data are not perfectly consistent, though the predictions are good enough from practical point of view. For example, Figure 22 shows experimental and simulated MP bands for coaxial line with inner diameter $\mathrm{D}=40 \mathrm{~mm}$ of outer conductor, outer diameter $\mathrm{d}=12.46$ $\mathrm{mm}$ of central conductor, impedance $\mathrm{Z}=70 \mathrm{Ohm}$ at $\mathrm{f}=1.3$ $\mathrm{GHz}$ of travelling wave (coaxial line of cold part of a variant of TTF-III power coupler). None of the simulation results fits perfectly experimental data. We will look for the reasons of this inconsistency again in the variations of SEY parameters. The MP dynamic is also complicated by the non-linear fields in coaxial lines. So, the additional factors such as impedance $\mathrm{Z}$ and frequency $\mathrm{f}$ will be considered. Standing wave ratio will be also considered.

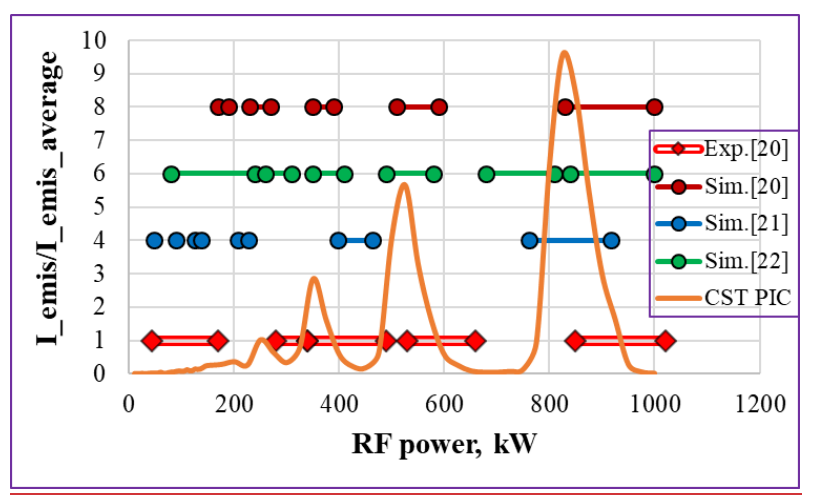

Figure 22: MP barriers in TTF-III power coupler. Discrepancy between the experiment and the various simulations is shown.

The model of coaxial line to be used is simple: it is a piece of line with two ports at the ends (see Fig.23). In transit time solver of CST both ports deliver power into the line and absorb the waves reflected from opposite sides. Different combinations of field amplitudes and phases in the ports produce a variety of waves of different standing wave ratio and amplitudes.

As a basic parameter to characterize a variety of the considered coaxial lines the parameter introduced in [23] for traveling wave will be used:

$$
S=\ln \left[(f \cdot D)^{4} \cdot Z\right],
$$

where $\mathrm{f}, \mathrm{D}$ and $\mathrm{Z}$ are operating frequency in $\mathrm{GHz}$, inner diameter of outer conductor in $\mathrm{mm}$ and impedance in $\mathrm{Ohm}$ respectively. For most of the simulations this parameter was chosen $\approx 20$, since for this value excitation of more MP barriers is possible (see Fig.23). Other values of A were taken from some practical cases.

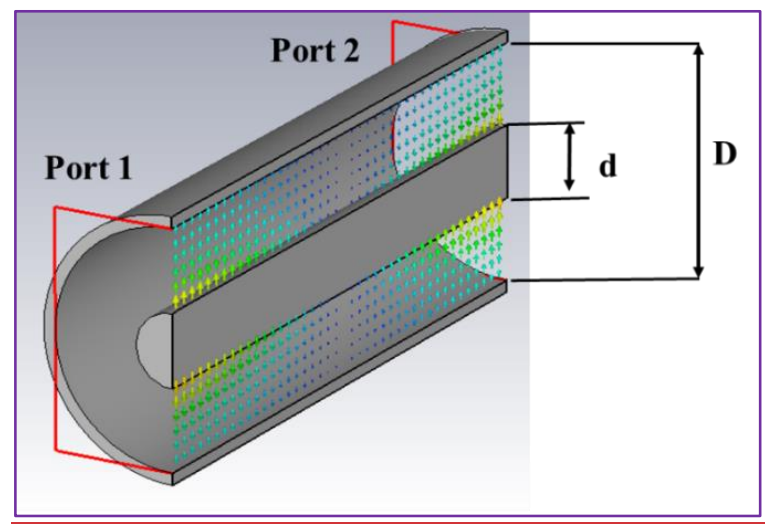

Figure 23: Snapshot of the traveling electric field in the model.

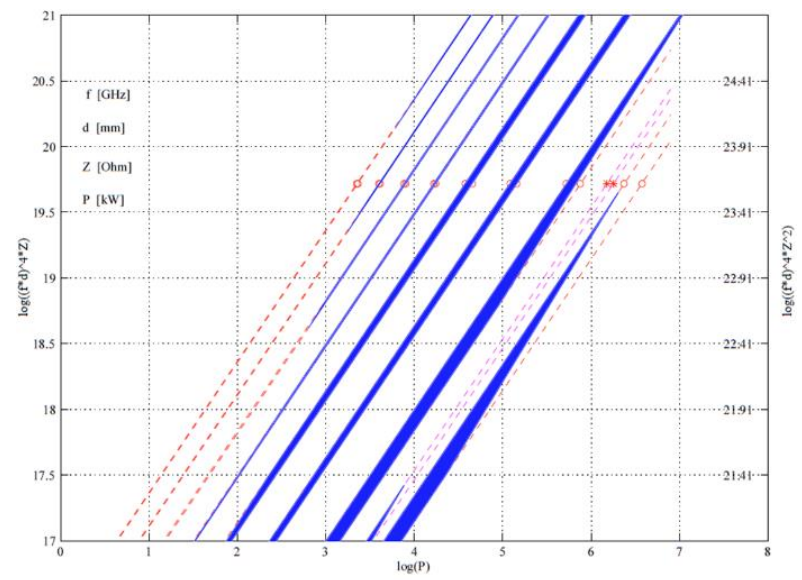

Figure 23: Multipactor susceptibility chart for coaxial lines [23]. The barriers indicated by circles correspond to $A=19.7$ (Group of the lines \#\#13-18 in Table 3)

Each value of parameter $S$ can be obtained with different combinations of $\mathrm{f}, \mathrm{D}$ and $\mathrm{Z}$. That was presumed a source of MP pattern variety in the coaxial lines along with different SEY parameters. For quick reference all combinations of the used coaxial line parameters are given in Table 3. The frequencies in the table are design ones, in some simulations they were different.

Table 3

\begin{tabular}{|l|l|l|l|l|l|}
\hline Line \# & $S$ & $\mathrm{Z}, \mathrm{Ohm}$ & $\mathrm{D}, \mathrm{mm}$ & $\mathrm{d}, \mathrm{mm}$ & $\mathrm{F}, \mathrm{GHz}$ \\
\hline 1 Perez & 20 & 103 & 72.9 & 12.7 & 0.65 \\
\hline 2 PIP-II & 11.94 & 35.82 & 49.8 & 27.18 & 0.1625 \\
\hline 3 TTF & 19 & 50 & 40 & 17.16 & 1.087 \\
\hline 4 & 19 & 60 & 40 & 14.5 & 1.0388 \\
\hline 5 & 19 & 75 & 40 & 11.25 & 0.9824 \\
\hline 6 & 19 & 95 & 40 & 8.03 & 0.926 \\
\hline 7 & 19 & 120 & 40 & 5.26 & 0.873 \\
\hline 8 & 19 & 50.7 & 29.035 & 12.456 & 1.4975 \\
\hline 9 & 19 & 50.7 & 40 & 17.16 & 1.087 \\
\hline 10 & 18.93 & 50.7 & 50 & 21.45 & 0.853 \\
\hline
\end{tabular}




\begin{tabular}{|l|l|l|l|l|l|}
\hline 11 & 18.94 & 50.7 & 60 & 25.974 & 0.716 \\
\hline 12 & 18.94 & 50.7 & 70 & 30.03 & 0.6091 \\
\hline 13 & 19.7 & 40 & 42.31 & 21.52 & 1.3 \\
\hline 14 & 19.7 & 50 & 40 & 17.16 & 1.3 \\
\hline 15 & 19.7 & 60 & 38.232 & 13.87 & 1.3 \\
\hline 16 & 19.7 & 75 & 36.16 & 10.18 & 1.3 \\
\hline 17 & 19.7 & 95 & 34.08 & 6.843 & 1.3 \\
\hline 18 & 19.7 & 120 & 32.15 & 4.23 & 1.3 \\
\hline Line \# & $S$ & Z, Ohm & D, $\mathrm{mm}$ & $\mathrm{d}, \mathrm{mm}$ & $\mathrm{F}, \mathrm{GHz}$ \\
\hline
\end{tabular}

\section{Impact of location of max SEY.}

The impact of collision energy value $\mathrm{W}_{\mathrm{m}}$ at which secondary emission is maximal was studied with SEY of material1.49@200,1.49@300 and1.49@400 in the coaxial lines \#1 and \#2. The simulations were performed at two frequencies of RF traveling wave: $162.5 \mathrm{MHz}$ for both lines and $650 \mathrm{MHz}$ for line \#2. The results of the simulations are presented in Fig.24-26.

No significant influence of this parameter on the MP barriers of $1^{\text {st }}$ order was found. The width and locations of the barriers are practically the same. Only the intensities and shapes of the barriers are slightly different. Interesting that this result is the same for different types of MP - one side MP in the line \#1 and two side MP in the line \#2.

This absence of effect may be explained by the fact that with active space charge an energy of collision is typically less than $\mathrm{W}_{\mathrm{m}}$, so a weak effect comes from the collisions closer to $\mathrm{W}_{1}$, where the difference between SEY is not large, rather than from exact value of $\mathrm{W}_{\mathrm{m}}$.

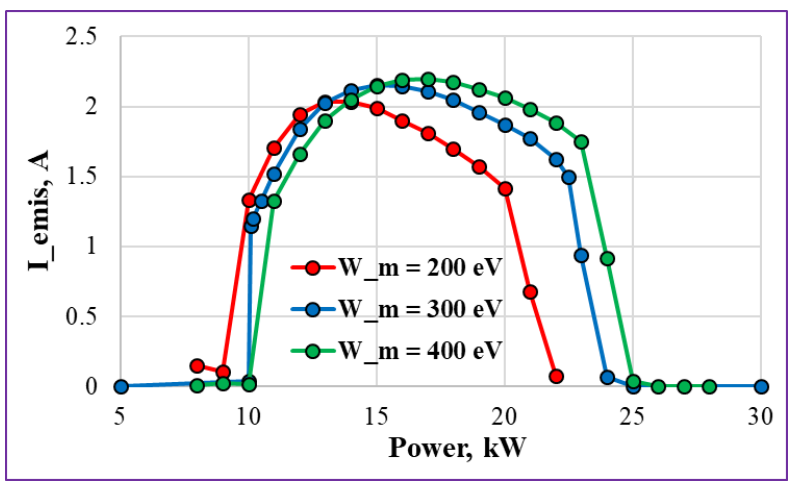

Figure 24: The barriers of one-point multipactor in the line \#1 at frequency $162.5 \mathrm{MHz}$.

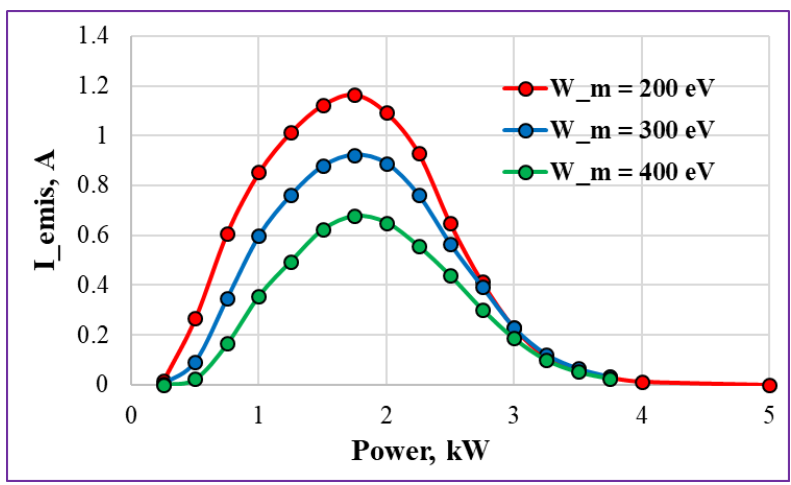

Figure 25: The barriers of two-point multipactor in the line \#2 at frequency $162.5 \mathrm{MHz}$.

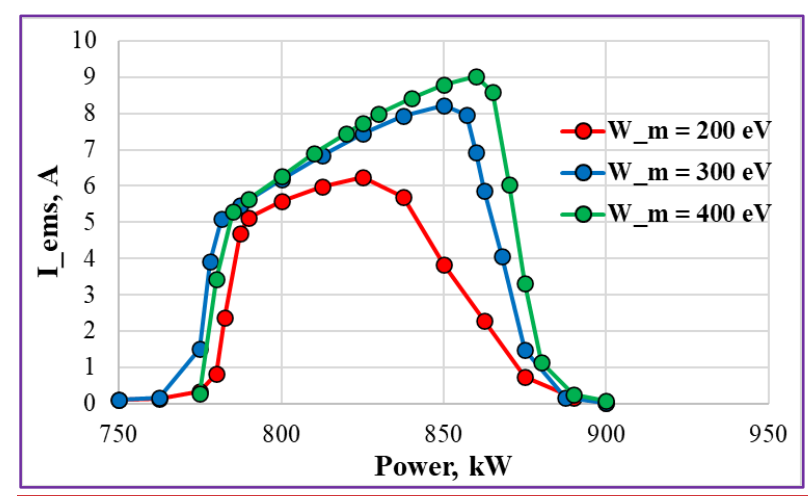

Figure 26. The barriers of one-point multipactor in the line $\# 1$ at frequency $650 \mathrm{MHz}$.

\section{$M P$ in transition from $T W$ to $S W$, and "electric" and "magnetic" $M P$}

Transition from TW to SW in short coaxial lines (much shorter than wavelength) is not recognized by MP dynamic, since the amplitude modulation and phase shift are negligible along sufficiently short line. The MP barriers in short lines are determined only by electric field strength between electrodes (of course if other conditions are met). A field level may be different at the same input power, depending on combination of amplitude and phase of reflected wave.

In the long lines, which length is comparable with wavelength, MP dynamic is more variable, the barriers and their locations in the lines depend on amplitude and phase of reflected wave. The effects of the reflected wave amplitude and phase are well known and shown in Fig.27 and 28. Different amplitudes of reflection create modulated waves with different depth of modulation, while different phases of reflection move crests and troughs of wave along line.

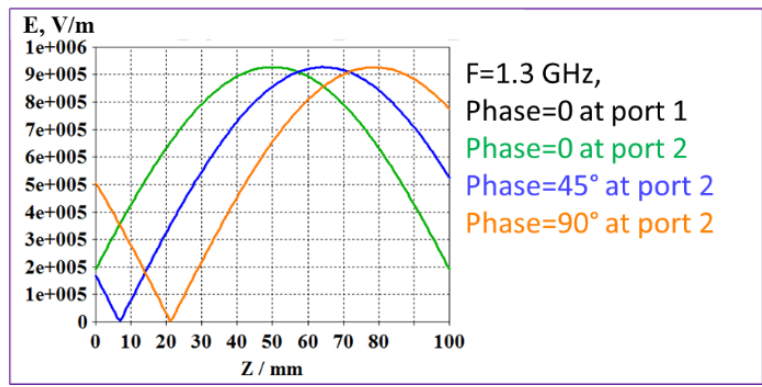

Figure 27: Electric field distributions for different phases of fully reflected wave $(\rho=1)$.

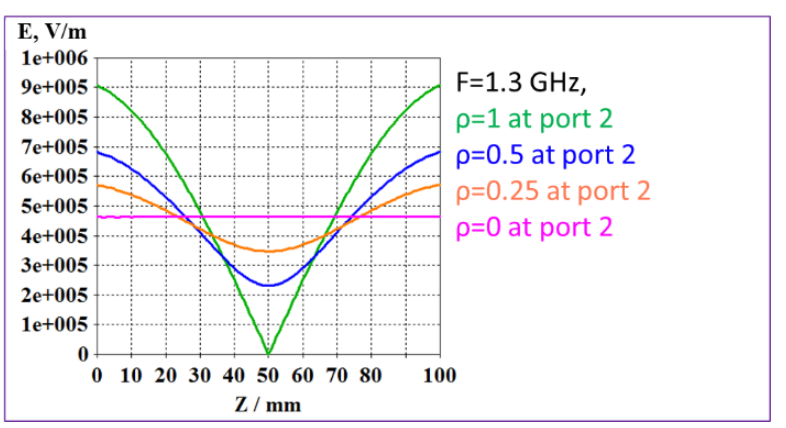

Figure 28: Electric field distribution for different reflections $\rho$ and fixed phase of reflected wave. 
Interesting effect can be observed as the reflection coefficient increases from 0 to 1 - the multipactor splits into so called "electric" and "magnetic" processes. Of course, the electrons are still accelerated by the electric field, and the terms only indicate the locations of MP- either around location of maximal electric field or around location of maximal magnetic field of modulated wave. The discussion on the physics of this effect is out of the scope of these notes, some insight can be found in $[21,23,24]$. Below there are the results of simulation of the "electric-magnetic" splitting in the coaxial line $\# 2$.

The simulations of MP of both kinds were performed in two short pieces of coaxial line \#2 to see their behaviour separately. Phases of reflection wave in the models were set to have maximum of electric field in one model and minimum of electric field in the other, the reflection coefficient and therefore the amplitude modulation were variable in both models (Fig, 29,30)

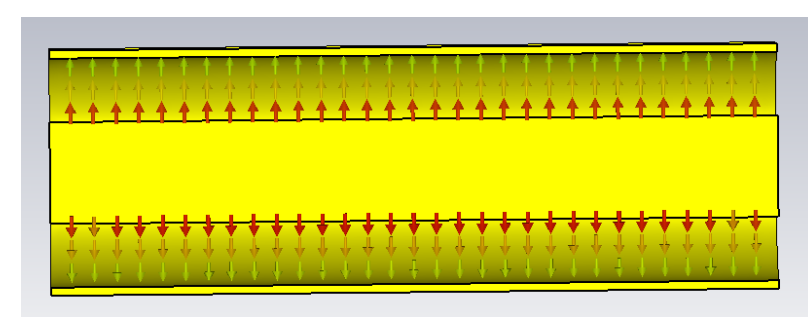

Figure 29: Electric field distribution in the model to simulate "electric" MP, $\rho=0.95$. Amplitude modulation practically is not detectable in such a short line even for almost SW field distribution.

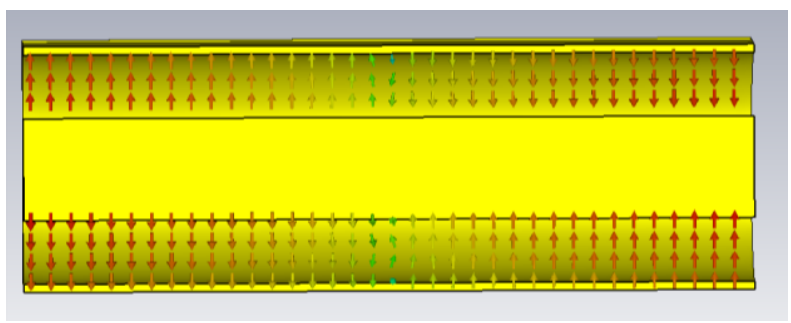

Figure 30: Electric field distribution in the model to simulate "magnetic" MP, $\rho=0.98$.

The evolution of MP barriers while reflection coefficient is changing from 0 to 1 (i.e., the traveling wave is transforming to standing one) are shown in Fig. 31 and 32 for both models.

The Fig. 33 shows how the MP power levels (centres of the barriers) of electric and magnetic barriers are moving relatively to each other with increasing of reflection coefficient. At high reflections ( $\rho>0.8$ in this case) the field modulation is deep and the area of field strength favourable for "magnetic" multiplication process around electric field minimum shrinks. As a result, "magnetic" MP starts at lower field level that provides more shallow modulation and compensates this spatial reduction of MP area.

In the long lines comparable with wavelength both "electric" and "magnetic" barriers can be excited close to each other in terms of input power. Therefore, the two barriers may overlap or be separated depending on reflection coefficient. When a reflection coefficient is low and field amplitude modulations is not very deep, MP continuously migrates from "electric" to "magnetic" location with increasing of input power (see Fig. 34) forming a single broad barrier. At each power level the MP in this barrier is a mix of "electric' and "magnetic" types in different proportions.

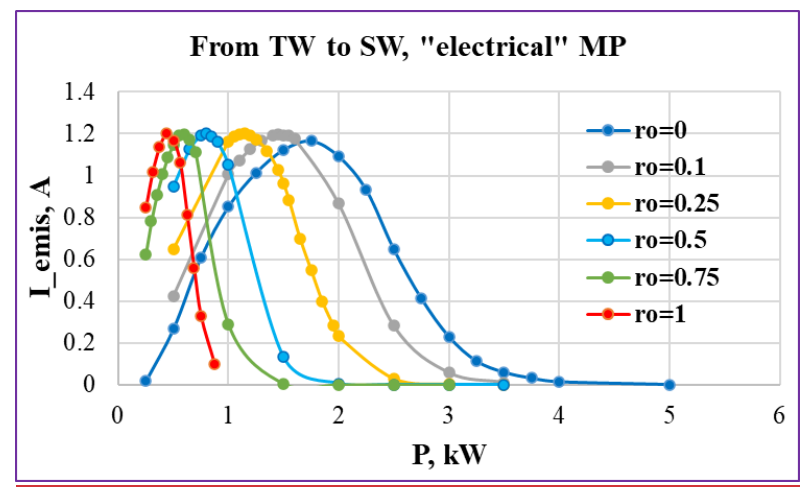

Figure 31: The "electrical" MP barriers for different reflection coefficient. Note the theoretical ratio of powers of $4: 1$ for $\mathrm{TW}(\rho=0)$ and SW $(\rho=1)$ barriers.

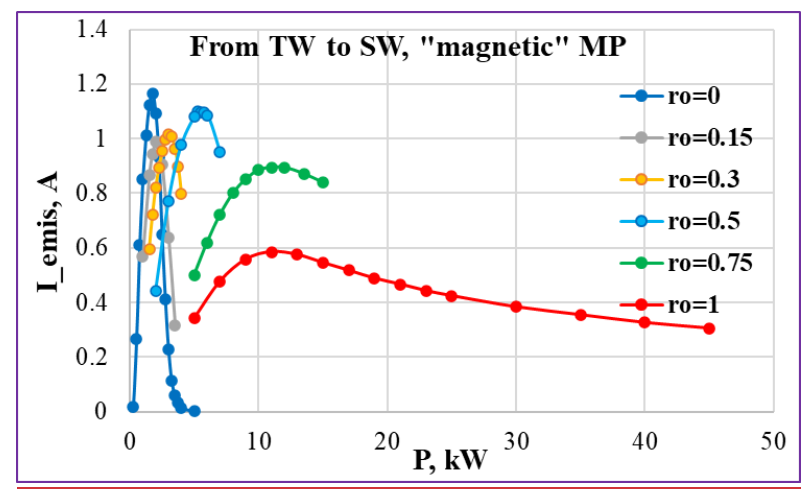

Figure 32: The "magnetic" MP barriers for different reflection coefficient.

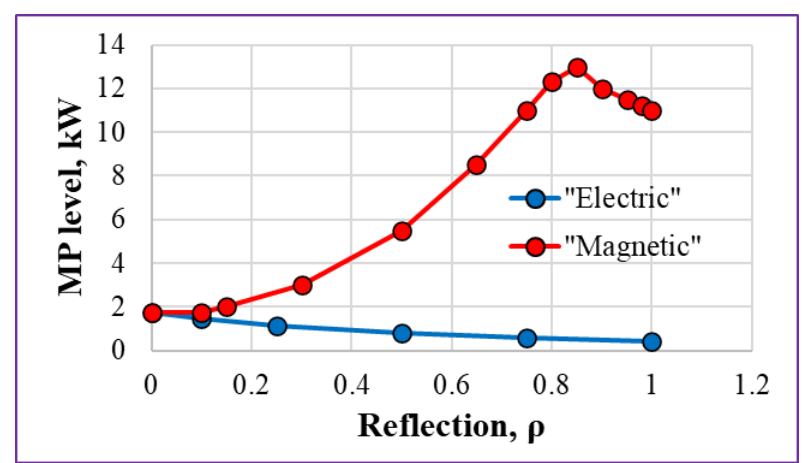

Figure 33: "Magnetic" and "electric" MP levels as functions of reflection coefficient.

The case of almost standing wave $(\rho=0.85)$ is shown in Fig.35. It is still continuous MP barrier, though it is weak at higher powers, so most likely it cannot be observed in practice. Nevertheless, the MP barrier in the long coaxial lines is expected wider at any reflection than simple theories predict due to the migration of MP along line. The plot in Fig.36a explains a migration of of discharge location in the long line at different power levels and reflections. The 
pictures of the MP location migration in the long line are shown in Fig.36b.

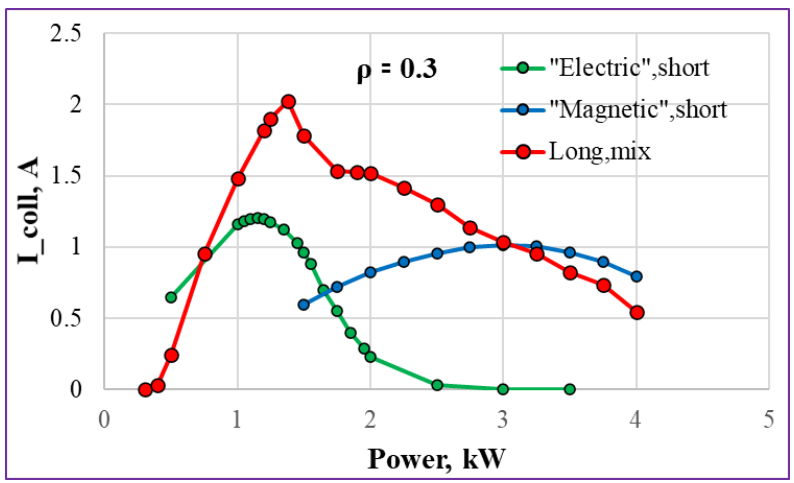

Figure 34: MP barrier in the long line $\# 2$ at $\rho=0.3$. The barriers for the short line at the same $\rho$ are given for comparison.

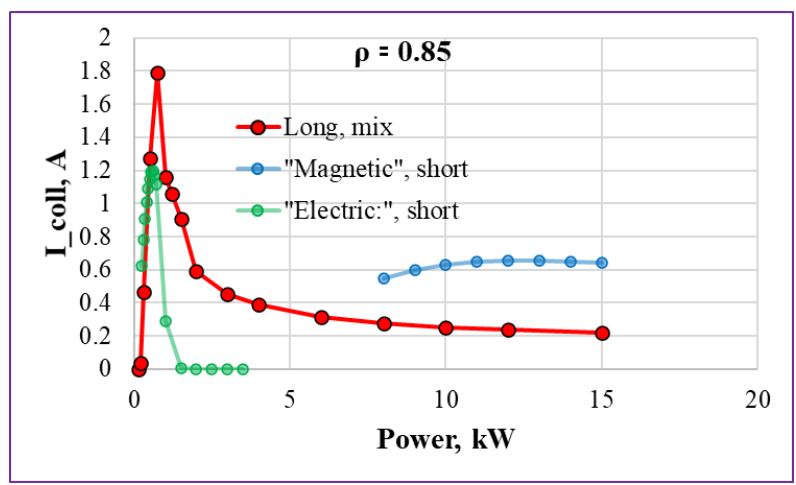

Figure 35: MP barrier in the long line $\# 2$ at $\rho=0.85$. The barriers for the short line at the same $\rho$ are given for comparison.

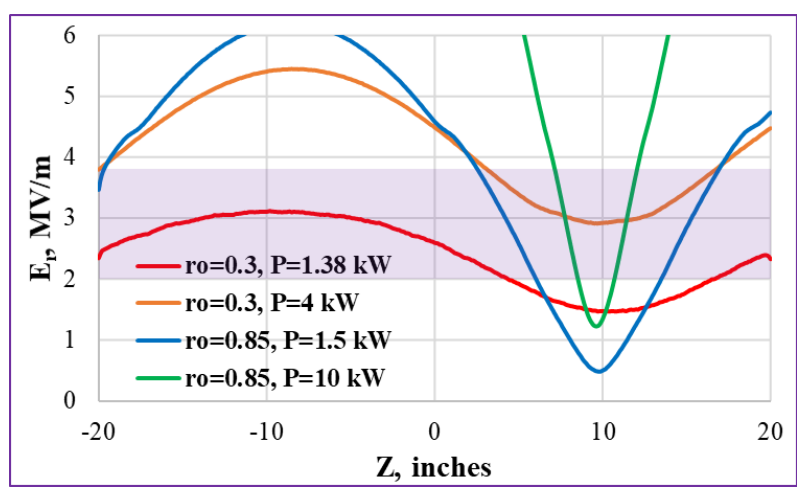

Figure 36a: Distributions of radial electric field amplitude $\mathrm{E}_{\mathrm{r}}$ on the central conductor at different input powers and reflections. Multipactor band is shown in violet.

\section{Impact of initial energy of secondary electrons}

The traveling wave simulations were performed in two coaxial lines with different most probable initial energy of secondary electrons $(\mathrm{Te}=0.1 \mathrm{eV}, 1 \mathrm{eV}$ and $7.5 \mathrm{eV})$ as it was done for parallel plates. The coaxial lines \#3 and \#4 with different impedances of $50 \mathrm{Ohm}$ and $120 \mathrm{Ohm}$ were used in these simulations. Secondary emission function 1.49@200 was assigned to the wall material. In the line \#3 the distance between outer and central electrodes of $17 \mathrm{~mm}$ is small enough at frequency $1 \mathrm{GHz}$, so the multipactor in this line is partially two side one. Therefore, the result of simulations for this line is similar to the case of parallel plates (see Fig.37 and compare to Fig.12). On the other hand, the radial electric field gradient, which dynamically acts as a returning force, is strong enough to provide one side multipactor on the wall of outer conductor. This mixed multipacting is well indicated by emission currents from the conductors (see Fig.38). The emission current from outer conductor is higher because it is generated by both one-side and two-side multipacting, while emission from the central conductor is due only to two-side process.

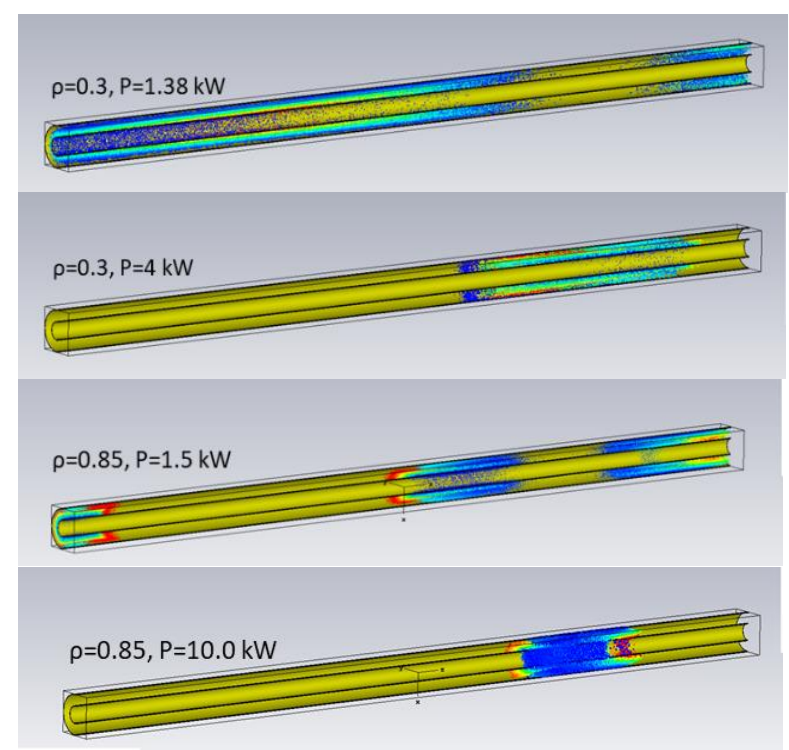

Figure 36b: The snapshots of multipacting in the long lines with the parameters corresponding to Fig.36a.

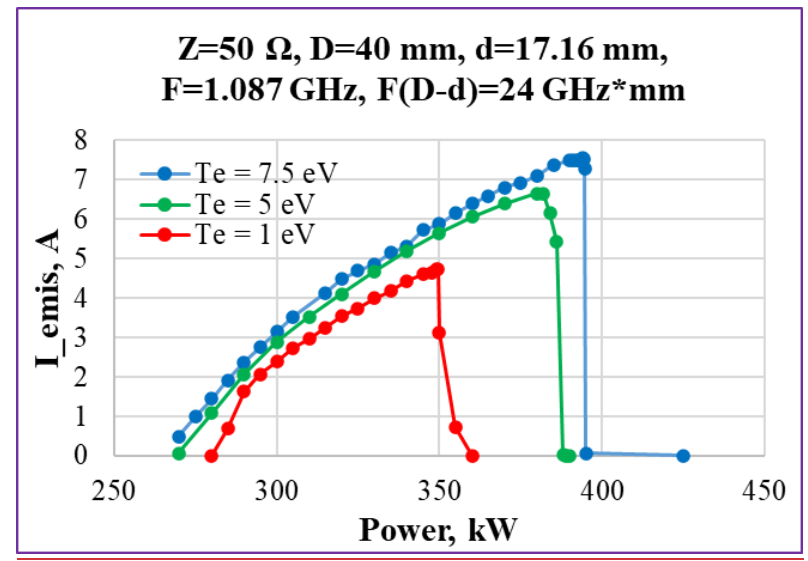

Figure 37: MP barriers in the line \#3 for different most probable emission energies of material.

In the line \#4 the MP is one side on the outer conductor, which is more typical for coaxial lines. In one side multipacting the particles fly two times longer between collisions (full RF period), than in case of two side MP (time of flight is half of RF period). So, the effect of the initial emission velocity is stronger for lines of higher impedances, and the shift of the barriers for different initial energy is more expressed (see Fig.39). 


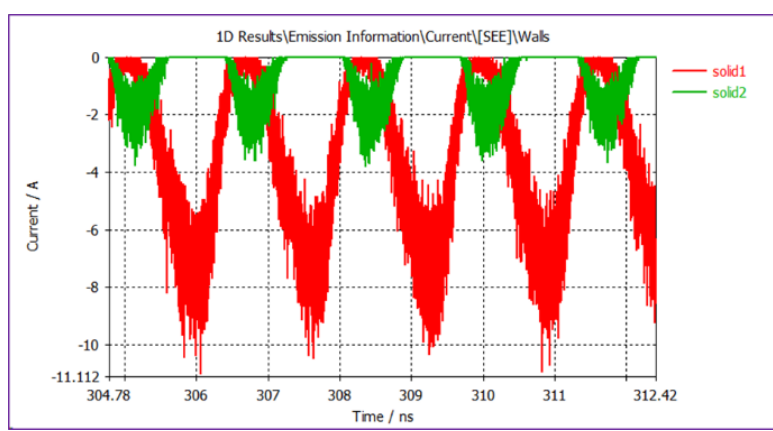

Figure 38: Emission currents from the outer conductor (red) and the inner (green) during multipacting in the line \#3.

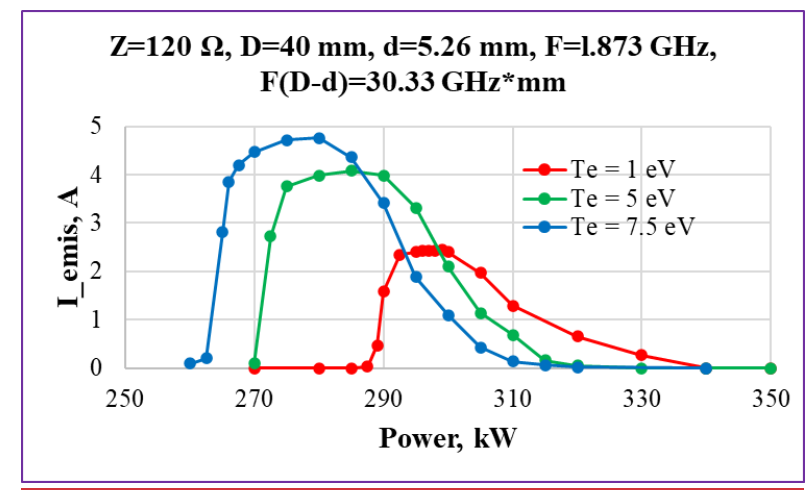

Figure 39: MP barriers in the line \#4 for different most probable emission energies of material.

\section{Impact of variable impedance with constant $A$}

In this set of simulations, the parameter $S=19$ was constant and the diameter of outer conductor $\mathrm{D}$ was fixed at 40 $\mathrm{mm}$. Impedance $\mathrm{Z}$ was variable, while frequency $\mathrm{f}$ and diameter of central conductor $d$ were adjusted accordingly to keep $S$ constant (lines \#\#3-7). From the susceptibility chart in Fig.23 first order MP was expected at input power of $295 \mathrm{~kW}(S=19=>\mathrm{P} \approx \ln (5.6875)=295 \mathrm{~kW})$.

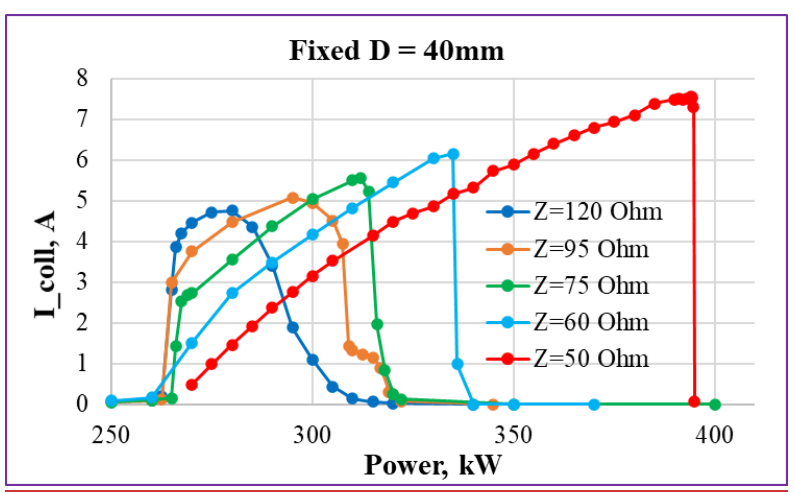

Figure 40: Transformation of MP barrier shapes in dependance on coaxial line impedance.

This way of keeping parameter $S$ constant results in gradual decreasing of the gap between outer and central conductors with decreasing Z. MP gradually transforms from one side mode to two side mode and the shape and the location of barrier change because of that (see Fig.40).
The mix of two MP modes in $50 \mathrm{Ohm}$ line is already shown in Fig.38. The figure shows emission currents from outer conductor and central conductor separately. It should be noticed that intensities of both modes are approximately equal, but amplitude of emission from outer conductor is higher because it is the sum of both modes as it was mentioned above.

\section{Impact of variable $D$ and $f$ with constant $S$}

In this set of simulations other way to keep $S=19$ constant was tested: the impedance was fixed at $50.7 \mathrm{Ohm}$, while frequency fand diameters of the conductors were adjusted accordingly to keep A constant (the lines \#\#9-12). With this approach the multiplication product $\mathrm{f} \times(\mathrm{D}-\mathrm{d})$ also remains constant.

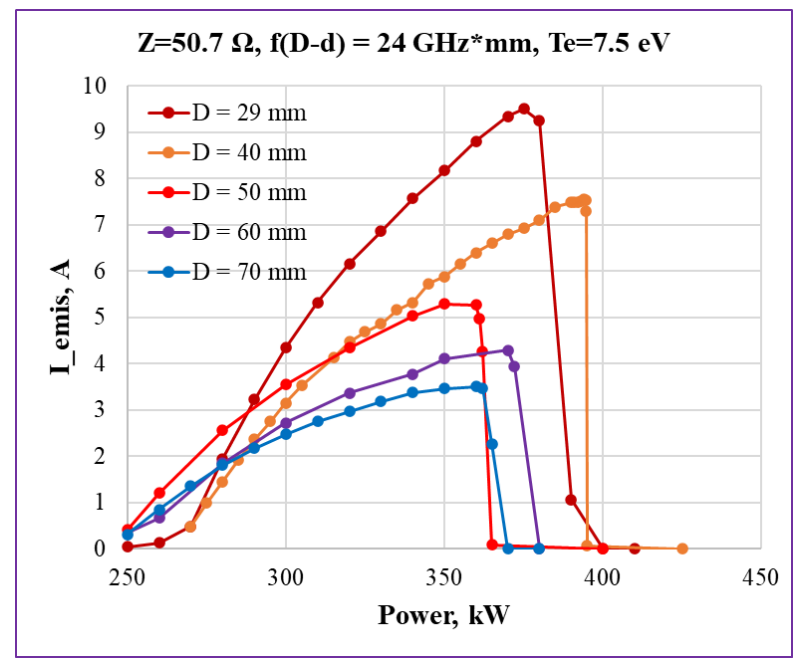

Figure 41: Transformation of MP barrier shapes in dependence on coaxial line outer diameter.

The results of simulations are shown in Fig.41. The plot suggests that $f \times(D-d)$, which is fundamental scaling parameter for parallel plates, and it works in similar way for coaxial lines (at least for this popular impedance of $50 \Omega$ ): namely, the shapes and intensities of MP barriers differ significantly, while the barrier locations are practically the same.

\section{Constant frequency}

In practice operating frequency almost never is a free parameter. It is determined by overall design goals, fixed, and cannot be changed. Therefore, the task is to evaluate and determine other parameters of a coaxial line to meet technical and physical requirements. In this set of simulations frequency was constant and equal to $1.3 \mathrm{GHz}$ parameter $S=19.7$ was also constant, while impedance $Z$ varied from 40 to 120 Ohm (lines\#\#13-18). Accordingly, the product $\mathrm{f} \times(\mathrm{D}-\mathrm{d}), \mathrm{D}$ and $\mathrm{d}$ were changing as shown in Fig.42. The MP barriers up to 3 order in this set of lines are shown in Fig.43.

In lines \#13 ( $Z=40 \mathrm{Ohm})$ and $\# 14(\mathrm{Z}=50 \mathrm{Ohm})$ the two side MP dominates because of smaller gap between outer and central electrodes, so the MP barriers in these lines are 
broader, have different shape and shifted toward upper boundary of MP barrier of 560-710 kW defined by susceptibility chart shown in Fig.23. For higher $Z$ the results are close to each other, look more similar and located near low boundary of the mentioned MP barrier. Other tendency is that when the gap between outer and central conductors increases the barriers get narrower due to the shrinking interval of stable phases.

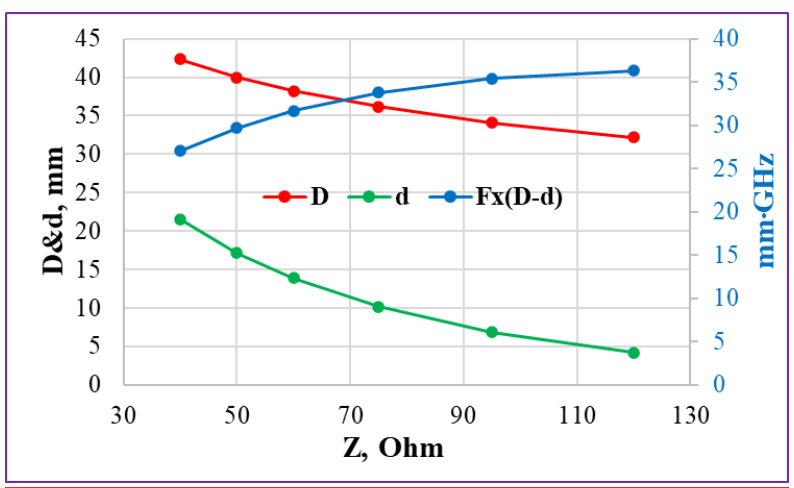

Figure 42: Diameters D and d, and the product f.(D-d) as functions of impedance $\mathrm{Z}$ while frequency $\mathrm{f}$ is kept constant.

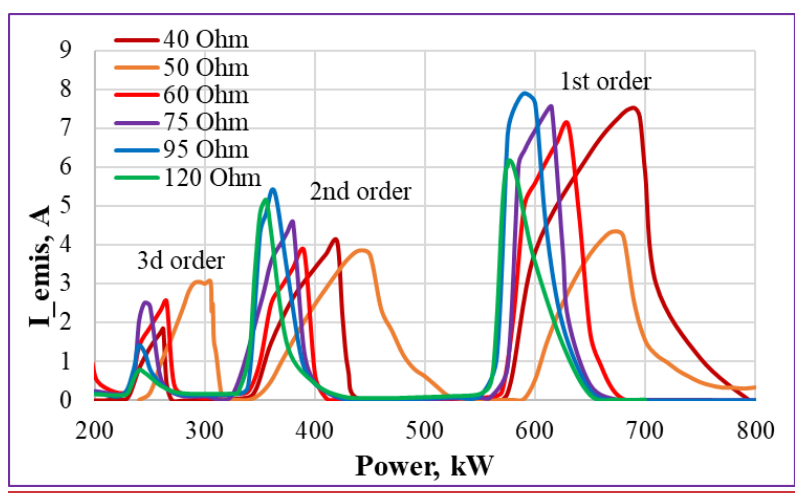

Figure 43: The multipactor barriers in the lines of different impedances at frequency $1.3 \mathrm{GHz}$.

\section{CONCLUSION}

In this work it was shown that the variations of material emission properties can change the multipactor characteristics significantly. The model used in PIC simulations are very simple, and they were widely used for analytical and numerical analyses of multipactor. But even in these simple structures the multipactor characteristics can be significantly different than classical theories predict. On the other hand, the basic knowledge how SEY parameters affect multipactor barriers may help to analyse multipactor in more complicated structures.

The parameters of the models and operational regimes such as coaxial line impedance, standing wave ratio, gap between parallel plates also add a complexity to the multipactor characteristics.

Multipactor is a space charge limited process, and space charge produces a global effect on the multipactor parameters, so it can dramatically change them. Therefore, it is highly recommended to include space charge effect in the simulations.

\section{REFERENCES}

[1] R. Seviour, "The Role of Elastic and Inelastic Reflection in Multipactor Discharges", IEEE Trans. on Electron Devices, vol.54, No 8, August, 2005

[2] G. Romanov, «Stochastic Features of Multipactor in Coaxial Waveguides for Travelling and Standing Waves», preprint PUB-11-003-TD, Fermilab, Batavia, IL 60510, USA, 2011.

[3] D. Proch, D. Einfeld, R. Onken, and N. Steinhauser, "Measurement of multipacting currents of metal surfaces in RF fields". WPQ24, IEEE Proceedings, PAC 95, 1776, 1996

[4] Л.В.Гришин и др., «Исследование вторично-эмиссионного СВЧ разряда при больших углах пролёта электронов», Труды Физического института им. Лебедева, 1997, том 92, стр, 82-131.

[5] Gennady Romanov, Paolo Berrutti and Timergali N. Khabiboulline, "Simulation of Multipacting in SC Low Beta Cavities at FNAL", in Proc. IPAC'15, Richmond, Virginia, USA, 2015, paper MOPMA018, pp. 579-581.

[6] C. W. Hoover, Jr., and R.K. Smither, "Secondary Electron Resonance Discharge Mechanism", Phys Rev. 98, 1149.(1955).

[7] A.J.Hatch and H.B.Williams, "Multipacting Modes of HighFrequency Gaseous Breakdown", The Physical Review, 2nd series, Vol.112, No.3, November 1, 1958.

[8] C. J. Lingwood et al, "Phase space analysis of multipactor saturation in rectangular waveguide", Physics of Plasmas 19, 032106 (2012).

[9] P. Berutti, T.Khabiboulline, G.Romanov, «Multipactor Discharge in the PIP-II Superconducting Spoke Resonators», Fermilab, Technical division, Technical note TD-16-005, 2016.

[10] V.A.Puntus, G.V.Romanov, "Multipacting in the Phase Beam Monitor at MMF", Proc. of EPAC98, Stockholm, 1998

[11] D.K.Callebout, "Secondary Electron Resonance Discharge: I. Steady State by Debunching”, Physica, Issue 7, July 1963, pp. 784-802.

[12] E. Sorolla and M. Mattes, "Multipactor saturation in parallel-plate waveguides", Phys. Plasmas 19, 072304 (2012)

[13] Liao Lang et al, "Multipacting Saturation in Parallel Plate and Micro-Pulse Electron Gun", Chinese Phys. C, Volume 39, Number 2, March 2014.

[14] Волков В.А., Ганичев Д.А., «Стационарное состояние вакуумного вторично-эмиссионного ВЧ разряда», Журн. техн. физ., 1982, т.52, №8, с. 1559-1563.

[15] Ганичев Д.А., Станский В.А., Фридрихов А.С., «Экспериментальное исследование эмиссии вторичных электронов на свервысоких частотах», Изв. АН СССР, Сер. физ., 1971, т.35, №2, с. 268-269. 1982, т.52, №8, с. $1559-1563$

[16] S.Riyopoulos, "Multipactor saturation due to space-charge induced debunching", Physics of Plasmas 4, 1448 (1997).

[17] A.Sazontov et al, "Effect of emission velocity spread of secondary electrons in two-sided multipactor", Phys. Plasmas, 12053102,2005 
[18] R.Woo and A.Ishimaru, "A Similarity Principle for Multipacting Discharges", Journal of Applied Physics, Vol. 38, \#13, 1967.

[19] M. Siddiqi, R. Kishek, "Simulation of Ping-Pong Multipactor With Continuous Electron Seeding", in Proc. NAPAC'16, Chicago, IL, USA, 2017, paper MOPOB53, pp. 181-183.

[20] K.Ge et al, "Multipacting simulations of TTF-III power coupler components", in Proc. PAC07, Albuquerque, New Mexico, USA, 2007

[21] E.Somersalo et al, "Analysis of Multipacting in Coaxial Lines”, FAE08, PAC95, Dallas, Texas, USA, 1995

[22] H. Jenhani et al, "Studies on Input Couplers for Superconducting Cavities", THP077, Linac08, Victoria, BC, Canada, 2008

[23] E.Somersalo et al, "Computational Methods for Analyzing Electron Multipacting in RF Structures", Particle Accelerators, Vol. 59, pp. 107-141, 1998 